\title{
High glucose-induced fibronectin upregulation in cultured mesangial cells involves caveolin-1-dependent RhoA-GTP activation via Src kinase
}

\author{
YONGFU LU ${ }^{1}$, LIHONG TANG ${ }^{2}$, YIQIAO $\mathrm{LI}^{3}$ and QIANG HE ${ }^{3}$ \\ ${ }^{1}$ Department of Pathology, Zhejiang University School of Medicine, the First Affiliated Hospital; \\ ${ }^{2}$ Department of Clinical Laboratory, Hangzhou First People's Hospital, Hangzhou, Zhejiang 310003; \\ ${ }^{3}$ Department of Nephrology, Zhejiang Provincial People's Hospital, Hangzhou, Zhejiang 310014, P.R. China
}

Received May 14,2015; Accepted March 21, 2016

DOI: $10.3892 / \mathrm{mmr} .2016 .5312$

\begin{abstract}
Increasing evidence indicates that diabetes-mediated renal interstitial fibrosis through extracellular matrix (ECM) protein accumulation is an important event in the development of diabetic kidney disease (DKD), however, the underlying mechanism remains unclear.In the current study, it was observed that high levels of glucose (HG) time- and dose-dependently increased the production of the ECM protein, fibronectin (FN), in primary rat mesangial cells. Inhibition of the Rho pathway blocked HG-induced FN upregulation. HG-induced RhoA activation was prevented by inhibiting caveolae with filipin III or caveolin-1 siRNA and rescued by exogenous caveolin-1. HG also increased caveolin-1/Src association and activated Src kinase, whereas the inhibition of Src blocked RhoA activation and FN upregulation. Src-mediated phosphorylation of caveolin-1 on Y14 has also been implicated in signaling responses. Overexpression of the nonphosphorylatable caveolin-1 Y14A mutant prevented the HG-induced RhoA activation and FN upregulation. In conclusion, $\mathrm{HG}$-induced FN upregulation requires caveolae and caveolin-1 to interact with RhoA and Src kinases. Interference with Src/caveolin-1/RhoA signaling may provide novel mechanistic targets for the treatment of DKD.
\end{abstract}

\section{Introduction}

Diabetes-mediated renal interstitial fibrosis is an important event in the development of diabetic kidney disease (DKD) (1). High glucose (HG) promotes the excessive accumulation of extracellular matrix (ECM) proteins and expression of fibrotic

Correspondence to: Miss. Yiqiao Li or Dr Qiang He, Department of Nephrology, Zhejiang Provincial People's Hospital, 158 Shangtang Road, Hangzhou, Zhejiang 310014, P.R. China

E-mail: yiqiao626@163.com

E-mail: qianghe@zju.edu.cn

Key words: caveolin-1, RhoA, Src, fibronectin, high glucose factors in mesangial cells (MCs), which leads to subsequent diabetic renal dysfunction (2). A variety of signaling molecules and pathways are associated with mesangial matrix proliferation, including ras homolog family member A (RhoA), a small GTPase protein. RhoA enhances actin cytoskeleton reorganization and endothelial cell barrier permeability $(3,4)$. Several studies have suggested that RhoA and Rho kinases are critical in the pathogenesis of DKD through glomerular sclerosis and ECM deposition, however the precise mechanism remains poorly understood $(3,4)$.

The Rho GTPases RhoA, ras-related C3 botulinum toxin substrate 1 (Rac1) and cell division cycle 42 (Cdc42) are small molecular switches that contribute to the control of cell morphology and motility (5). RhoA is known to regulate actomyosin-based contractility and retraction through the Rho-kinase pathway, and is also associated with cell apoptosis and proliferation (6). Previous reports have observed that RhoA is localized to the plasma membrane microdomains, caveolae, in cardiomyocytes and endothelial cells (7). The activation of RhoA and its downstream mediator Rho-kinase is a crucial step of the strain-induced production and secretion of fibronectin (FN) matrix protein in MCs, which depends on functional caveolae (8).

Caveolae are a specialized subset of lipid rafts that are most abundant in terminally differentiated cell types (9). Caveolin-1, the principal residual protein of the caveolae structure, functions as a scaffolding protein and directly interacts with various signaling kinases (10). Endogenous caveolin-1 depletion is associated with reduced $v$-akt murine thymoma viral oncogene homolog (AKT) and extracellular regulated mitogen-activated protein kinase 1/2 (ERK1/2) phosphorylation, and impaired tube formation in vascular endothelial and smooth muscle cells (11). Additionally, high glucose has been demonstrated to alter the caveolar protein localization of RhoA, and increase the activation of phosphatidylinositol 3-kinase/Akt, mitogen-activated protein kinase (MAPK), and AMP-activated protein kinase signaling cascades in cardiomyocytes (12). However, the association of caveolae/caveolin-1 with HG-induced dysfunction of MCs has not been assessed. Therefore, the current study explored the potential function of caveolae in RhoA signaling activation by $\mathrm{HG}$ and its importance in ECM accumulation in MCs. 


\section{Materials and methods}

Cell culture. This study was approved by the ethics committee of Zhejiang Provincial People's Hospital (Hangzhou, China). A total of 10 Sprague-Dawley rats (weight, 230-250 g) were bred in the Zhejiang Key Laboratory of Experimental Animal and Safety Evaluation (Hangzhou, China). These rats were maintained in a room with controlled temperature $\left(22^{\circ} \mathrm{C}\right)$ and a reverse 12-h light/dark cycle. They were supplied with food and water ad libitum. Primary MCs were obtained from the glomeruli of the rats by differential sieving as previously described (13) and cultured in Dulbecco's modified Eagle's medium (DMEM) supplemented with $20 \%$ fetal calf serum (FCS; Invitrogen; Thermo Fisher Scientific,Inc., Waltham, MA, USA), streptomycin (100 $\mu \mathrm{g} / \mathrm{ml}$; Dalian Meilun Biotech Co., Ltd., Dalian, China) and penicillin (100 U/ml; Dalian Meilun Biotech Co., Ltd.) at $37^{\circ} \mathrm{C}$ in $95 \%$ air, $5 \% \mathrm{CO}_{2}$. Experiments were performed using cells between passages 6 and 15 . Following pre-incubation in DMEM supplemented with $0.1 \%$ FCS for $24 \mathrm{~h}$, the confluent MCs were treated with $\mathrm{HG}(40 \mathrm{mM}$; Sigma-Aldrich, St. Louis, MO, USA). Pharmacological inhibitors were purchased from Sigma-Aldrich and added at the following concentrations and times prior to HG stimulation: HL07, $10 \mu \mathrm{M}$ for $60 \mathrm{~min}$; filipin III, $2.5 \mu \mathrm{g} / \mathrm{ml}$ for $10 \mathrm{~min}$; and SU6656, $10 \mu \mathrm{M}$ for $30 \mathrm{~min}$.

Western blotting. The experiments for the immunoblotting were performed at least 3 times. MCs were lysed with radioimmunoprecipitation assay (Beyotime Institute of Biotechnology, Haimen, China) lysis buffer containing phenylmethanesulfonyl fluoride (Beyotime Institute of Biotechnology) protease inhibitor.Protein concentrations were determined using the bicinchoninic acid method (Beyotime Institute of Biotechnology, Haimen, China). Proteins from total cell lysates were resolved by $10 \%$ sodium dodecyl sulfate-polyacrylamide gel electrophoresis (SDS-PAGE), transferred to a polyvinylidene fluoride (PVDF) membrane, blocked in 5\% non-fat milk in Tris-buffered saline with Tween-20 (Sigma-Aldrich) at $25^{\circ} \mathrm{C}$ for $2 \mathrm{~h}$. The antibodies used mouse monoclonal anti-FN (cat. no. 610077; 1:5,000) and mouse anti-phospho-caveolin-1 Y14 (cat. no. 611339, 1:1,000) (BD Biosciences, Franklin Lakes, NJ, USA); mouse monoclonal anti-RhoA (cat. no. sc-418; 1:500; Santa Cruz Biotechnology, Inc., Dallas, TX, USA); mouse monoclonal anti-caveolin-1 (cat. no. 05-762 clone 7C8; 1:1,000; Upstate Biotechnology, Inc., Lake Placid, NY, USA); polyclonal phospho-SrcY416 (1:1,000; cat. no. 2102) and polyclonal Src (1:1,000; cat. no. 2109) (Cell Signaling Technology, Inc., Danvers, MA, USA); and mouse monoclonal anti- $\beta$-actin (cat. no. A5441; 1:5,000; Sigma-Aldrich). Actin and total RhoA were used as the internal reference to calculate the relative intensity of objective protein bands. Goat anti-mouse IRDye 680LT (926-68050) and goat anti-rabbit IRDye $800 \mathrm{CW}$ (925-32211) secondary antibodies (LI-COR Biotechnology, Lincoln, NE, USA) were diluted 1:20,000 with blocking buffer with $0.1 \%$ Tween- 20 and incubated in the dark at $25^{\circ} \mathrm{C}$ for $1 \mathrm{~h}$. Finally, the PVDF membrane was observed using the Odyssey Classic imager (LI-COR Biotechnology) and associated Image Pro analysis 3.1.4 software (Media Cybernetics Rockville, MD, USA).
RhoA pulldown assay. MCs were lysed in hypertonic buffer ( $30 \mathrm{mM}$ HEPES, $1.5 \mathrm{mM} \mathrm{MgCl} 2,450 \mathrm{mM} \mathrm{NaCl}$, $0.3 \mathrm{mM}$ EDTA and $10 \%$ glycerol), and RhoA-GTP was immunoprecipitated from the cleared lysate with $25 \mu$ glutathione-S-transferase-tagged rhotekin-RhoA-binding domain bound to glutathione-agarose (Cytoskeleon, Inc., Denver, CO, USA). Beads were washed, and the immunoprecipitate was resolved with $15 \%$ SDS-PAGE. Membranes were probed with monoclonal anti-RhoA antibody (1:500, Santa Cruz Biotechnology, Inc.). The lysate (40 $\mu \mathrm{g})$ was also probed for RhoA to ensure equal loading across conditions.

Transfection. Rat MCs were transiently transfected with caveolin-1 siRNA (sc-29942; Santa Cruz Biotechnology, Inc.) using the X-tremeGene siRNA transfection reagent (Roche Applied Science, Penzberg, Germany) according to the manufacturer's protocol. The transfected MCs were assayed 24-48 h post-transfection.

The rat caveolin-1 coding sequence was amplified from MC cDNA. Briefly, MC RNA was extracted using TRIzol reagent (Takara Bio Inc., Otsu, Japan) and $2 \mu \mathrm{g}$ was reverse-transcribed with Superscript II (Takara Bio Inc.) according to the manufacturer's instructions. Samples were incubated at $95^{\circ} \mathrm{C}$ for $3 \mathrm{~min}$, followed by 30 cycles of $95^{\circ} \mathrm{C}$ for $1 \mathrm{~min}, 57^{\circ} \mathrm{C}$ for $1 \mathrm{~min}$ and $72^{\circ} \mathrm{C}$ for $1 \mathrm{~min}$. The resulting cDNA was used for semiquantitative PCR amplification of caveolin-1, using $\beta$-actin as an internal control. The sequence was inserted into the vector pEGFP-C1 using the restriction sites HindIII-BamHI (6084-1, Clontech Laboratories Inc., Mountain View, CA, USA) with an NH2-terminal enhanced green fluorescent protein (EGFP) for use as caveolin-1 rescue. Using the plasmid as a template, Y14 of caveolin-1 was mutated to alanine with the QuikChange II Site-Directed Mutagenesis kit (Agilent Technologies, Santa Clara, CA, USA). Templates were incubated at $95^{\circ} \mathrm{C}$ for $1 \mathrm{~min}$, then for 20 cycles of $95^{\circ} \mathrm{C}$ for $1 \mathrm{~min}, 65^{\circ} \mathrm{C}$ for $4 \mathrm{~min}$, and $68^{\circ} \mathrm{C}$ for $12 \mathrm{~min}$. The mutagenic primers were as follows: Sense, $5^{\prime}$-TCGGAGGGACA TCTCGCCACCGTTCCCATCCG-3' and anti-sense, 5'-CGGA TGGGAACGGTGGCGAGATGTCCCTCCGA-3'. Rat MCs were transfected with empty vector or pEGFP-Cav-1Y14A using Lipofectamine 3000 (Invitrogen; Thermo Fisher Scientific, Inc.). At 18-24 h post-transfection, the medium was changed to $0 \%$ FCS and the experiment continued.

Statistical analysis. Statistical analysis was performed using GraphPad Prism 6.0 (GraphPad Software, Inc., La Jolla, CA, USA). Comparing several groups was conducted using analysis of variance with Fisher's Least Significant Difference test. $\mathrm{P}<0.05$ was considered to indicate a statistically significant difference. Data represent the mean \pm standard error of three independent experiments.

\section{Results}

HG-induced FN upregulation requires RhoA/Rho kinase activation. Exposure of rat glomerular MCs to HG was demonstrated to increase the protein expression level of intracellular FN at all time points compared with the $0 \mathrm{~h}$ control $(\mathrm{P}<0.05$; Fig. 1A). The current study also analyzed FN secretion following HG stimulation. The levels of extracellular FN 
A
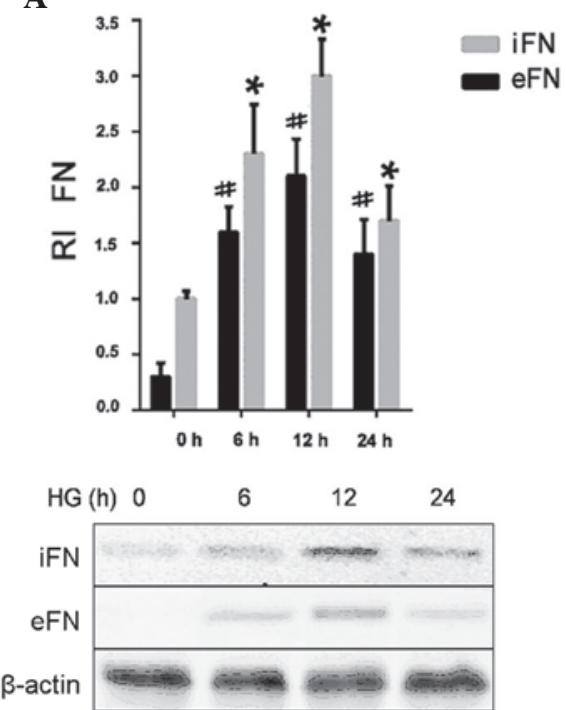

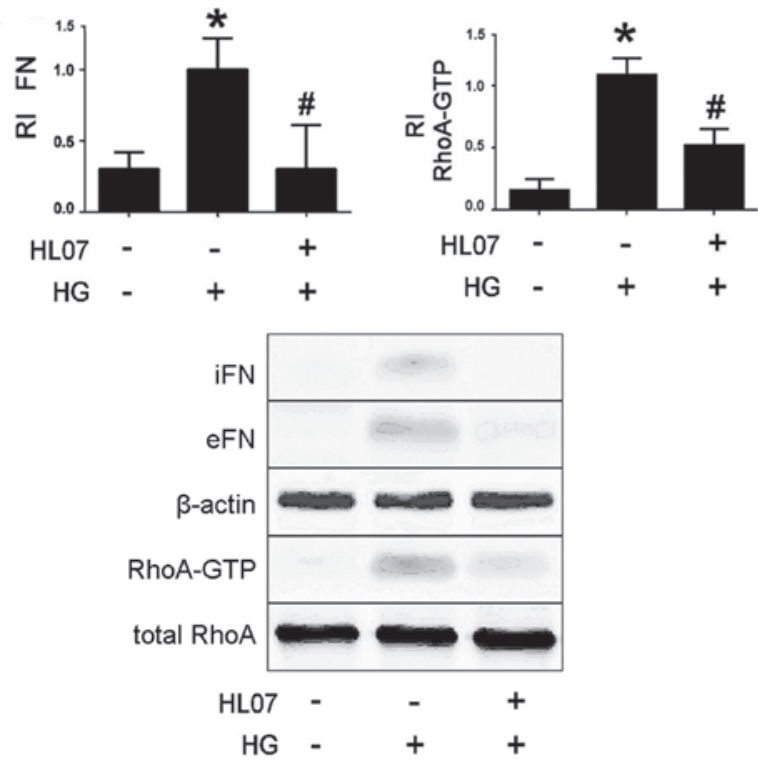

Figure 1. HG induces a significant increase in FN protein levels via RhoA/Rho kinase activation. Data represent the mean \pm standard error of three independent experiments. (A) MCs were treated for the indicated times with $40 \mathrm{mM} \mathrm{HG}$, then FN protein levels were assessed by western blotting, with $\beta$-actin used as a loading control. ${ }^{*} \mathrm{P}<0.05$ vs. iFN control $(0 \mathrm{~h}) ;{ }^{*} \mathrm{P}<0.05$ vs. eFN control $(0 \mathrm{~h})$. (B) MCs were pretreated with a RhoA kinase inhibitor HL07 (10 $\left.\mu \mathrm{M}, 60 \mathrm{~min}\right)$ and subsequently treated with $40 \mathrm{mM} \mathrm{HG}$ for $6 \mathrm{~h}$, FN protein and RhoA-GTP protein levels were assessed by western blotting, with $\beta$-actin used as the internal reference to calculate the relative intensity of $\mathrm{FN}$ and total RhoA used as loading control. ${ }^{*} \mathrm{P}<0.05$ vs. control (no $\mathrm{HG}$ treatment); ${ }^{\sharp} \mathrm{P}<0.05$ vs. $\mathrm{HG}$ treatment. HG, high glucose; FN, fibronectin; RI, relative intensity; iFN, intracellular FN; eFN, extracellular FN; RhoA-GTP, Ras homolog family member A-GTP; MC, mesangial cell. Data are representative of three independent experiments.

were increased significantly following stimulation at all time points, compared with the $0 \mathrm{~h}$ control $(\mathrm{P}<0.05$; Fig. $1 \mathrm{~A})$. The increased FN secretion was accompanied by the activation of RhoA and an increase in total RhoA in rat MCs, compared with untreated control cells ( $\mathrm{P}<0.05$; Fig. 1B). To investigate the activity of RhoA on HG-induced FN secretion, rat MCs were pretreated with RhoA inhibitor, HL07. It was observed that HL07 treatment decreased the FN protein levels compared with HG treatment $(\mathrm{P}<0.05)$, demonstrating that $\mathrm{RhoA}$ is required for $\mathrm{HG}$-induced $\mathrm{FN}$ upregulation in MCs (Fig. 1B).

HG induces caveolin-1 upregulation and phosphorylation via Src kinases. RhoA has been has been demonstrated to localize to the caveolae in MCs (13). Therefore, the current study explored whether HG influences caveolin-1 expression in rat MCs. It was observed that caveolin-1 Y14 phosphorylation was increased following 5, 10 and 30 min HG treatment in a time-dependent manner, compared with the 0 min time-point $(\mathrm{P}<0.05$; Fig. 2A). There was a trend toward increased total caveolin-1 protein levels following HG treatment, however, this was not statistically significant ( $\mathrm{P}>0.05$; Fig. 2A).

Src kinases are the only kinases known to phosphorylate caveolin-1 at Y14 (3). The present study confirmed that HG-induced caveolin-1 Y14 phosphorylation was mediated by $\mathrm{Src}$ in rat MCs. As illustrated in Fig. 2B and C, HG treatment resulted in the autophosphorylation of Src Y416 and increased association between Src and caveolin-1 compared with untreated control cells. Treatment of MCs with SU6656 Src inhibitor prevented the HG-induced caveolin-1 Y14 phosphorylation, whereas, it exhibited no effect on HG-induced upregulation of caveolin-1 protein expression (Fig. 2D).
HG-induced RhoA activation requires intact caveolae. The current study examined the effects of caveolar disruption on HG-induced RhoA activation and FN upregulation. The membrane-permeable agent filipin III and caveolin-1 siRNA were used to perturb the formation of caveolae. It was observed that filipin III and caveolin-1 siRNA prevented HG-induced RhoA activation compared with HG-only treatment (Fig. 3A). The present study additionally investigated whether the effects of caveolar disruption could be reversed by expressing a non-targetable caveolin-1 complementary DNA (rescue). Exogenous caveolin-1 expression reversed the effects of the caveolin-1 siRNA on RhoA activation in MCs $(\mathrm{P}<0.05$; Fig. 3A). However, it exhibited no significant effect on RhoA activity inhibition in MCs pretreated with filipin III and HG. These data suggest that HG-induced RhoA activation requires caveolar structural integrity in MCs.

Caveolin-1 Y14A prevents $H G$-induced RhoA activation and FN secretion. Caveolin-1 Y14 phosphorylation by Src kinases is associated with RhoA activation (3). As the current study had observed that the HG-induced RhoA activation in MCs requires caveolae, a caveolin-1 Y14A mutant in which the tyrosine is replaced by the non-phosphorylatable alanine was constructed to establish the definitive role of caveolin-1 Y14 phosphorylation in this setting (Fig. 3B). HG-induced FN secretion was abrogated in MCs expressing the caveolin-1 Y14A mutant (Fig. 4A). Caveolin-1 Y14A failed to interact with RhoA and effectively prevented HG-induced RhoA activation (Fig. 4B and C). These results demonstrate the importance of caveolin-1 Y14 phosphorylation for the $\mathrm{HG}$-induced RhoA activation and increased FN secretion in MCs. 
A

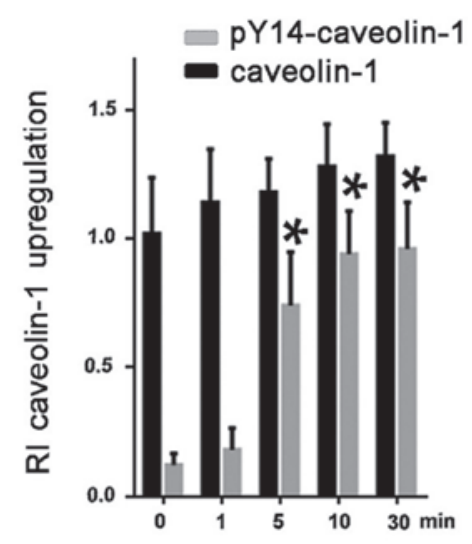

B
C

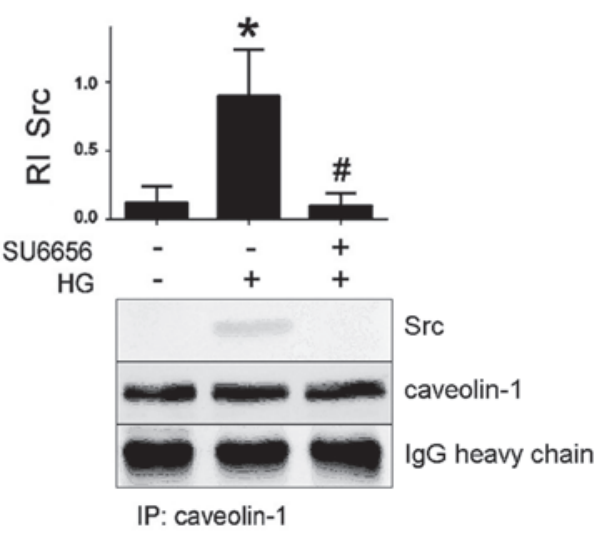

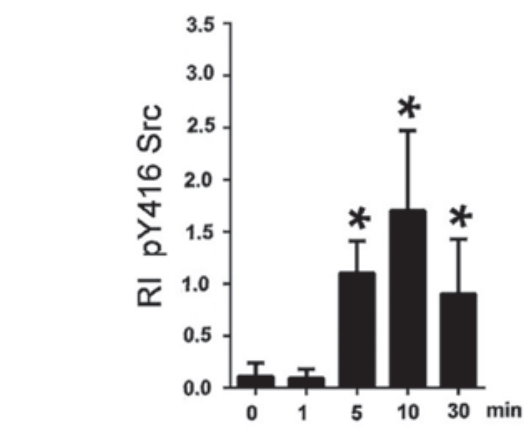
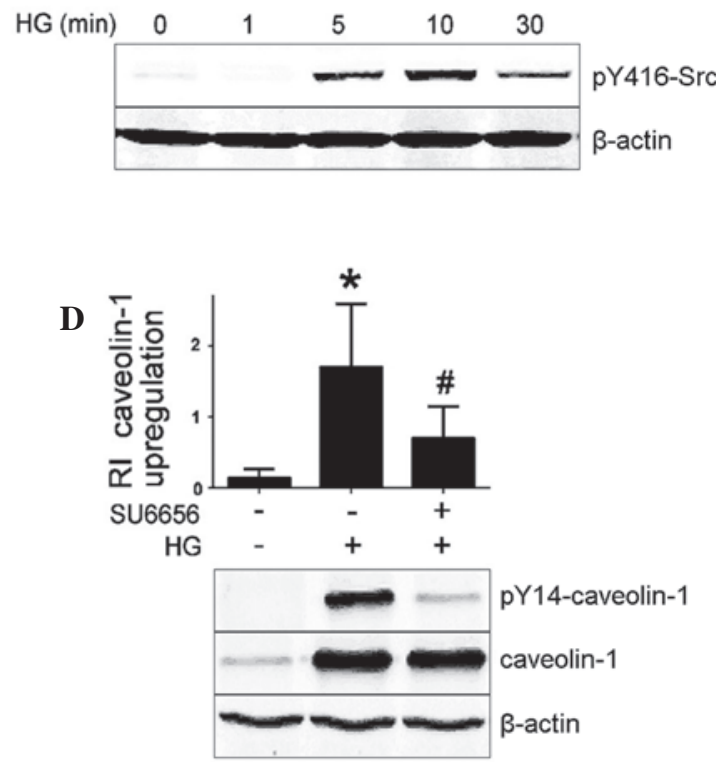

Figure 2. HG-induced caveolin-1 protein level and caveolin-1 Y14 phosphorylation upregulation via Src kinase activation. Data represent the mean \pm standard error of three independent experiments. (A) MCs were treated for the indicated times with HG and caveolin-1 protein level and phosphorylation at Y14 were assessed by western blotting. ${ }^{*} \mathrm{P}<0.05$ vs. pY416-caveolin- 1 control $(0 \mathrm{~min})$. (B) MCs were treated for the indicated times with HG and Src protein level and phosphorylation at Y416 were assessed by western blotting. "P<0.05 vs. control (0 min). (C) MCs were pretreated with a Src kinase inhibitor SU6656 (10 $\mu \mathrm{M}$, $30 \mathrm{~min}$ ) and treated with $40 \mathrm{mM} \mathrm{HG}$ for $10 \mathrm{~min}$, IP of caveolin-1 was conducted, and its association with $\mathrm{Src}$ was assessed by western blotting. "P<0.05, vs. control (no treatment); ${ }^{\#} \mathrm{P}<0.05$ vs. HG treatment. (D) MCs were pretreated with a Src kinase inhibitor SU6656 (10 $\left.\mu \mathrm{M}, 30 \mathrm{~min}\right)$ and treated with $40 \mathrm{mM}$ HG for 10 min, and caveolin-1 protein level and caveolin-1 Y14 phosphorylation were assessed by western blotting. " $\mathrm{P}<0.05 \mathrm{vs}$. control (no treatment); ${ }^{~} \mathrm{P}<0.05$, vs. HG treatment. $\beta$-actin was used as loading control for western blotting to calculate the relative intensity of Src and caveolin-1. Data are representative of three independent experiments. HG, high glucose; MC, mesangial cell; RI, relative intensity; IP, immunoprecipitation.

\section{Discussion}

The induction of caveolin-1 expression by high concentrations of glucose is associated with the upregulation of FN expression in rat MCs (14). The current study observed that HG treatment results in a physical association between caveolin-1 and RhoA. It was additionally demonstrated that the caveolar integrity is important in allowing caveolin-1/RhoA interaction and activation, which depend on the Src-mediated phosphorylation of caveolin-1 at Y14 $(8,15,16)$. These data suggest that the disruption of caveolin-1 action may attenuate the renal tissue deterioration induced by HG.

A previous study demonstrated that HG treatment stimulated caveolar-localized protein tyrosine phosphorylation in the isolated plasma membrane of various cell types, and resultant MAPK/ERK activation was dependent on intact caveolae (17). The current study demonstrated that RhoA activation requires caveolae integrity, which further characterizes these microdomains as important transducers of Src/phospho-caveolin-1/RhoA signaling (8). The data of the present study also indicated that HG treatment regulated the caveolin-1 protein levels and the caveolar structural formation in rat MCs. However, the mechanisms of caveolar localization and how interaction with caveolin-1 facilitates RhoA activation remains to be further elucidated.

Caveolin-1 Y14 N-terminal phosphorylation was first identified in $v$-Src-transformed cells, and the caveolin-1 phosphorylation response appeared to be cell type and stimulus-specific $(3,13,18,19)$. In the current study, HG treatment stimulated a sustained increase in Src Y416 autophosphorylation and caveolin-1 Y14 phosphorylation, which subsequently enabled RhoA activation. Using SU6656 Src 
A

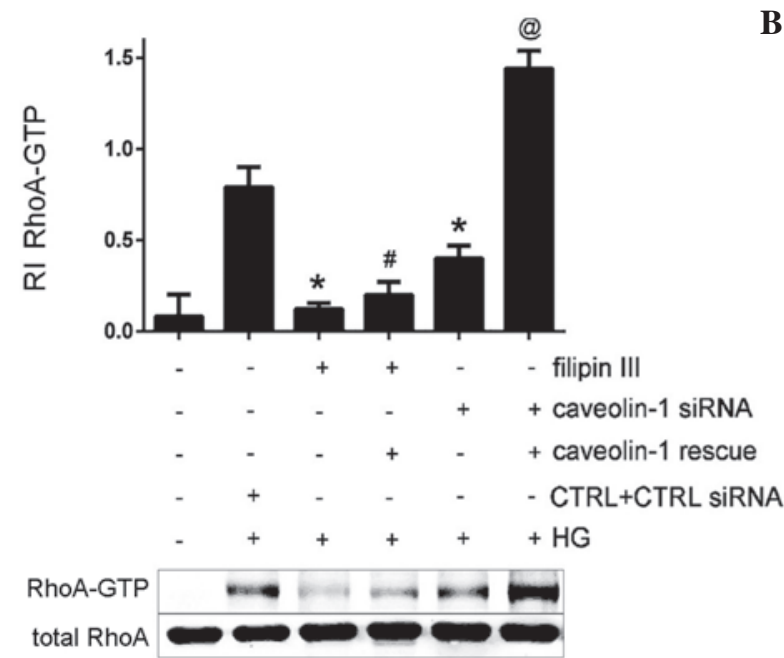

B

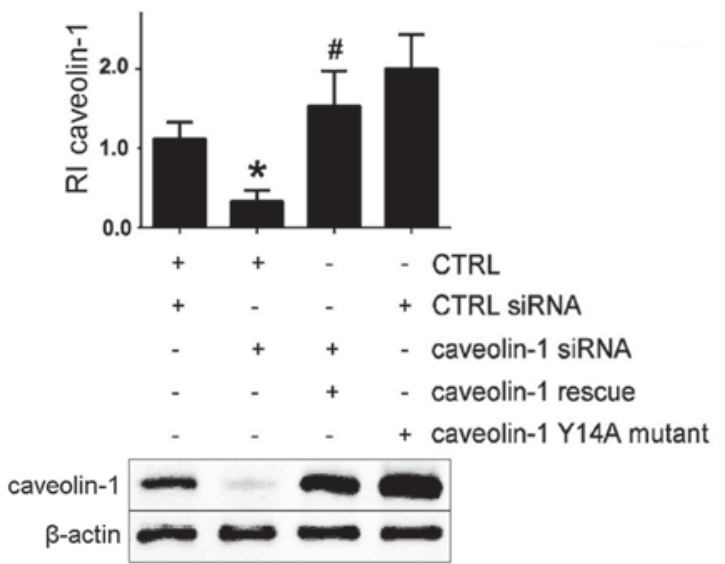

Figure 3. Caveolar disruption prevents HG-induced RhoA activation. Data represent the mean \pm standard error of three independent experiments. (A) MCs expressing caveolin-1 siRNA or exogenous caveolin-1 rescue were pretreated with a caveolar-disrupting agent (filipin III, $2.5 \mu \mathrm{g} / \mathrm{ml}, 10 \mathrm{~min}$ ) and $40 \mathrm{mM}$ HG. RhoA-GTP protein level was assessed by western blotting, with total RhoA used as loading control to calculate the relative intensity of RhoA-GTP. " $\mathrm{P}<0.05$ vs. control (no filipin III or caveolin-1 siRNA); ${ }^{*} \mathrm{P}>0.05$ vs. filipin III; ${ }^{\circledR} \mathrm{P}<0.05$ vs. caveolin- 1 siRNA treatment. (B) MCs were treated with caveolin-1 siRNA or exogenous caveolin-1 rescue, and caveolin-1 protein level was assessed by western blotting, with $\beta$-actin used as loading control to calculate the relative intensity of caveolin- $1 .{ }^{~} \mathrm{P}<0.05$ vs. control (CTRL siRNA treatment). ${ }^{*} \mathrm{P}<0.05$ vs. caveolin- 1 siRNA treatment. HG, high glucose; MC, mesangial cell; RI, relative intensity; RhoA, Ras homolog family member A. Data are representative of three independent experiments.

A

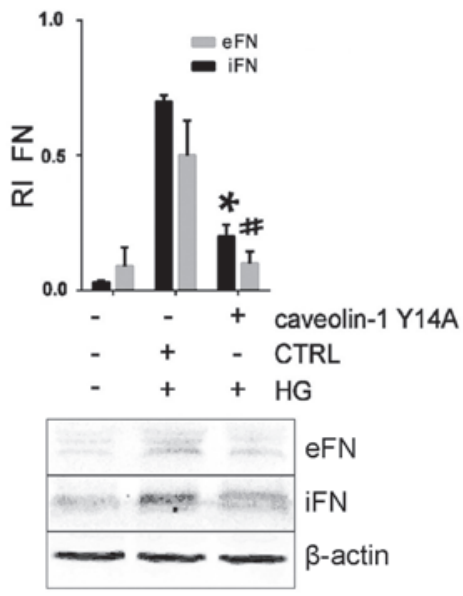

B

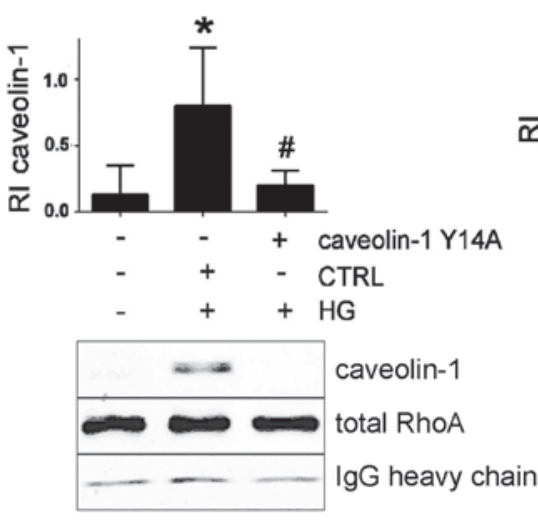

IP: RhoA
C

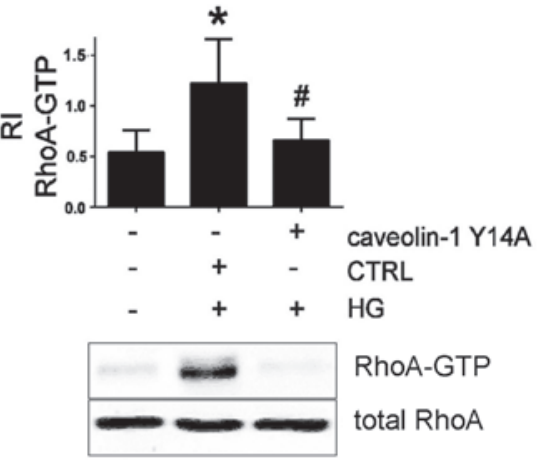

Figure 4. Caveolin-1 Y14A mutant prevents HG-induced FN upregulation and RhoA activation, assessed by western blotting. Data represent the mean \pm standard error of three independent experiments. MCs were pretreated with caveolin-1 Y14A mutant and treated with HG. (A) FN protein levels, with $\beta$-actin used as a loading control to calculate the RI. ${ }^{*} \mathrm{P}<0.05$ vs. iFN HG + CTRL siRNA; ${ }^{\text {P }}<0.05$ vs. eFN HG + CTRL siRNA. (B) Caveolin-1 was immunoprecipitated, and its association with RhoA was assessed. ${ }^{*} \mathrm{P}<0.05$ vs. control (no HG); ${ }^{\text {"P }}<0.05$ vs. HG + CTRL siRNA. (C) RhoA-GTP protein level, with total RhoA used as loading control to calculate the RI of RhoA-GTP. ${ }^{*} \mathrm{P}<0.05$ vs. control (no HG); ${ }^{\text {P }}<0.05$ vs. HG + CTRL siRNA. HG, high glucose; FN, fibronectin; eFN, extracellular FN; iFN, intracellular FN; RI, relative intensity; CTRL, control siRNA; RhoA, Ras homolog family member A; MC, mesangial cell; IP, immunoprecipitation. Data are representative of three independent experiments.

inhibitor and the non-phosphorylatable mutant caveolin-1 Y14A, the present study demonstrated that caveolin-1 Y14 phosphorylation, mediated by Src, is required for the downstream FN production and secretion in MCs $(20,21)$. However, the initial mechanisms involved in HG-dependent phosphorylation of caveolin-1 by Src kinase and the relationships between these signaling components induced by HG remain obscure, and require further research.
In summary, the present study highlights the importance of caveolin-1 and caveolae in HG-induced ECM accumulation. The requirement for Src kinase activity demonstrates the importance of caveolin-1 Y14 phosphorylation in the subsequent activation of RhoA and fibronectin upregulation. These findings serve to elucidate a mechanism that may contribute to the progression of DKD and control of caveolin-1 in renal tissue, and may be an alternative treatment 
possibility for delaying the renal dysfunction induced by excessive HG.

\section{Acknowledgements}

The present study was supported by the Natural Science Foundation of Zhejiang Province, China (no. LQ15H050003) and the National Science Foundation of China (no. 30670810).

\section{References}

1. Zou X, Zhang XX, Liu XY, Li R, Wang M, Wu WJ, Sui Y and Zhao HL: Renal kallikrein activation and renoprotection after dual blockade of renin-angiotensin system in diet-induced diabetic nephropathy. J Diabetes Res 2015: 310645, 2015.

2. Lo CS, Chang SY, Chenier I, Filep JG, Ingelfinger JR, Zhang SL and Chan JS: Heterogeneous nuclear ribonucleoprotein F suppresses angiotensinogen gene expression and attenuates hypertension and kidney injury in diabetic mice. Diabetes 61: 2597-2608, 2012.

3. Wu SZ, Peng FF, Li JL, Ye F, Lei SQ and Zhang BF: Akt and RhoA activation in response to high glucose require caveolin-1 phosphorylation in mesangial cells. Am J Physiol Renal 306: F1308-F1317, 2014.

4. Xie X, Peng J, Chang X, Huang K, Huang J, Wang S, Shen X, Liu $\mathrm{P}$ and Huang H: Activation of RhoA/ROCK regulates NF- $\kappa \mathrm{B}$ signaling pathway in experimental diabetic nephropathy. Mol Cell Endocrinol 369: 86-97, 2013.

5. Mao Y and Finnemann SC: Regulation of phagocytosis by Rho GTPases. Small GTPases 6: 88-99, 2015.

6. PerryNA,VitoloMI,MartinSSandKontrogianni-KonstantopoulosA: Loss of the obscurin-RhoGEF downregulates RhoA signaling and increases microtentacle formation and attachment of breast epithelial cells. Oncotarget 5: 8558-8568, 2014.

7. Kawamura S, Miyamoto S and Brown JH: Initiation and transduction of stretch-induced RhoA and Racl activation through caveolae: Cytoskeletal regulation of ERK translocation. J Biol Chem 278: 31111-31117, 2003.

8. Peng F, Wu D, Ingram AJ, Zhang B, Gao B and Krepinsky JC: RhoA activation in mesangial cells by mechanical strain depends on caveolae and caveolin-1 interaction. J Am Soc Nephrol 18: 189-198, 2007.

9. Stary CM, Tsutsumi YM, Patel PM, Head BP, Patel HH and Roth DM: Caveolins: Targeting pro-survival signaling in the heart and brain. Front Physiol 3: 393, 2012.

10. Shankar J, Boscher C and Nabi IR: Caveolin-1, galectin-3 and lipid raft domains in cancer cell signalling. Essays Biochem 57: 189-201, 2015.
11. Kang JW and Lee SM: Impaired expression of caveolin-1 contributes to hepatic ischemia and reperfusion injury. Biochem Biophys Res Commun 450: 1351-1357, 2014.

12. Balteau M, Van Steenbergen A, Timmermans AD, Dessy C, Behets-Wydemans G, Tajeddine N, Castanares-Zapatero D, Gilon P, Vanoverschelde JL, Horman S, et al: AMPK activation by glucagon-like peptide-1 prevents NADPH oxidase activation induced by hyperglycemia in adult cardiomyocytes. Am J Physiol Circ Physiol 307: H1120-H1133, 2014.

13. Wu T, Zhang B, Ye F and Xiao Z: A potential role for caveolin-1 in VEGF-induced fibronectin upregulation in mesangial cells: Involvement of VEGFR2 and Src. Am J Physiol Renal Physiol 304: F820-F830, 2013.

14. Zhang Y, Peng F, Gao B, Ingram AJ and Krepinsky JC: High glucose-induced RhoA activation requires caveolae and PKC $\beta 1$-mediated ROS generation. Am J Physiol Renal Physiol 302: F159-F172, 2012.

15. Jansen M, Pietiainen VM, Polonen H, Rasilainen L, Koivusalo M, Ruotsalainen U, Jokitalo E and Ikonen E: Cholesterol substitution increases the structural heterogeneity of caveolae. J Biol Chem 283: 14610-14618, 2008.

16. Radeva G, Perabo J and Sharom FJ: P-Glycoprotein is localized in intermediate-density membrane microdomains distinct from classical lipid rafts and caveolar domains. FEBS J 272: 4924-4937, 2005.

17. Karlsson M, Thorn H, Danielsson A, Stenkula KG, Ost A, Gustavsson J, Nystrom FH and Stralfors P: Colocalization of insulin receptor and insulin receptor substrate-1 to caveolae in primary human adipocytes. Cholesterol depletion blocks insulin signalling for metabolic and mitogenic control. Eur J Biochem 271: 2471-2479, 2004.

18. Jiao H, Zhang Y, Yan Z, Wang ZG, Liu G, Minshall RD, Malik AB and Hu G: Caveolin-1 Tyr14 phosphorylation induces interaction with TLR4 in endothelial cells and mediates MyD88-dependent signaling and sepsis-induced lung inflammation. J Immunol 191: 6191-6199, 2013.

19. Lee H, Volonte D, Galbiati F, Iyengar P, Lublin DM, Bregman DB, Wilson MT, Campos-Gonzalez Boumediene Bouzahza R, Pestell RG, Scherer PE and Lisanti MP: Constitutive and growth factor-regulated phosphorylation of caveolin-1 occurs at the same site (Tyr-14) in vivo: identification of a c-Src/Cav-1/Grb7 signaling cassette. Mol Endocrinol 14: 1750-1775, 2000.

20. Lee SH, Lee YJ, Park SW, Kim HS and Han HJ. Caveolin-1 and integrin betal regulate embryonic stem cell proliferation via p38 MAPK and FAK in high glucose. J Cell Physiol 226: 1850-1859, 2011.

21. Park JH, Ryu JM and Han HJ. Involvement of caveolin-1 in fibronectin-induced mouse embryonic stem cell proliferation: Role of FAK, RhoA, PI3K/Akt, and ERK 1/2 pathways. J Cell Physiol 226: 267-275, 2011. 